\title{
The Stochastic Geometry of Invasion Percolation
}

\author{
J. T. Chayes ${ }^{1, \star}$, L. Chayes ${ }^{1, \star}$, and C. M. Newman ${ }^{2, \star \star}$ \\ 1 Departments of Mathematics and Physics, Harvard University, Cambridge, MA 02138, USA \\ 2 Department of Mathematics, University of Arizona, Tucson, AZ 85721, USA
}

\begin{abstract}
Invasion percolation, a recently introduced stochastic growth model, is analyzed and compared to the critical behavior of standard $d$-dimensional Bernoulli percolation. Various functions which measure the distribution of values accepted into the dynamically growing invaded region are studied. The empirical distribution of values accepted is shown to be asymptotically unity above the half-space threshold and linear below the point at which the expected cluster size diverges for the associated Bernoulli problem. An acceptance profile is defined and shown to have corresponding behavior. Quantities related to the geometry of the invaded region are studied, including the surface to volume ratio and the volume fraction. The former is shown to have upper and lower bounds in terms of the above defined critical points, and the latter is bounded above by the probability of connection to infinity at the half-space threshold. Provided that the critical regimes of Bernoulli percolation possess their anticipated properties, as is known to be the case in two dimensions, these results verify numerical predictions on the acceptance profile, establish the existence of a sharp surface to volume ratio and show that the invaded region has zero volume fraction. Large-time asymptotics are analyzed in terms of the probability that the invaded region accepts a value greater than $x$ at time $n$. This quantity is shown to be bounded below by $h(x) \exp \left[-c(x) n^{(d-1) / d}\right]$ for $x$ above threshold, and to have an upper bound of the same form for $x$ larger than a particular value (which coincides with the threshold in $d=2$ ). For two dimensions, it is also established that the infinite-time invaded region is essentially independent of initial conditions.
\end{abstract}

\section{Introduction}

Invasion percolation is a stochastic growth model that was introduced and studied numerically by two independent groups $[1,2]$. (See also [28] for some related

* National Science Foundation Postdoctoral Research Fellows. Work supported in part by the National Science Foundation under Grant No. PHY-82-03669

$\star \star$ John S. Guggenheim Memorial Fellow. Work supported in part by the National Science Foundation under Grant No. MCS-80-19384 
ideas.) This is the first in a series of papers in which we analyze the mathematical aspects of invasion percolation. In this paper, we restrict attention to the simplest version of the model: invasion without trapping, as defined below. Invasion with trapping will be treated elsewhere [3].

The interest in invasion percolation is twofold:

1) The original applications $[1,2,4]$ concerned the study of fluid flow and transport properties in a random medium. However, unlike other models of this sort - such as standard Bernoulli percolation or first passage problems, which in general describe the passive diffusion of fluid particles - invasion percolation is used to describe the active displacement of an ambient fluid by a second fluid under pressure. The numerical simulations [4] motivated by the dynamical aspects of the model suggested another, initially surprising, application:

2) In a sense to be made precise below, invasion seems to reproduce, both qualitatively and quantitatively, standard Bernoulli percolation at threshold. It can therefore be used to study the critical behavior of the standard "static" percolation model.

The purpose of our work is to examine, and hopefully clarify the relationship between the dynamic and static models. We provide proofs of some of the numerically observed properties, and also several new properties which further substantiate the claim that invasion reproduces critical percolation. In order to put our results into context, let us first review the definition of the model and the relevant numerical and theoretical results.

\section{The Model and Previous Results}

For simplicity, we consider only the $d$-dimensional hypercubic bond lattice $\mathbb{B}_{d}$, which is, by definition, the set of all bonds between nearest neighbor pairs of sites on the site lattice $\mathbb{Z}^{d}$. (It should, however, be noted that the model is easily defined on more general graphs, and that our results extend to these in a straightforward fashion.) The first step is to assign independent random variables, uniformly distributed in $[0,1]$, to the bonds of $\mathbb{B}_{d}$. For a given realization on $\mathbb{B}_{d}$, one generates the invaded regions, which are an evolving sequence of connected bond clusters. The algorithm for generating the $(n+1)^{\text {st }}$ region from the $n^{\text {th }}$ is simply to select the bond on the boundary of the $n^{\text {th }}$ region with the smallest value. As discussed in the appendix (for $d=2$ ), the zeroth region (or "starting set") may be chosen arbitrarily, provided that it is finite. For the purposes of this paper, we will always start from the origin of coordinates.

It should be rather clear that the above procedure is equivalent to one in which random numbers are assigned to bonds only when (and if) they become attached to the boundary of the invaded region. (For obvious reasons, it is this setup which is studied numerically.) Thus, using the origin as a starting set, the first step is to assign values to the $2 d$ bonds surrounding the origin and to select the one with the lowest value. This bond is now the invaded region. Next, values must be assigned to the $2 d-1$ new bonds which have as an endpoint the other side (i.e., not the origin) of the chosen bond. From among the total of $4 d-2$ as yet unselected bonds which have already been assigned values, the one with the lowest value joins the first selected bond. These two bonds now constitute the invaded region, and the process continues. 
It is important to realize that, in the above procedure, new values are assigned only to those bonds which have not been previously assigned a value. For example, the second invaded bond described above is selected from a total of $4 d-2$ bonds, $2 d-1$ of them having "old" values, and $2 d-1$ of them "new." It is clear that the invaded region will have a statistical preference for the new bonds. Although this preference is somewhat offset at later stages by the preponderance of old bonds, it is nonetheless crucial to the stochastic geometry of the invaded region.

Let us now consider the evidence for the relationship between the invasion model and critical percolation. The numerical evidence $[4,27]$ falls into three classes:

1) In [4], a quantity called the acceptance profile at value $x$ and time $n$ was introduced and studied. In essence, this quantity measures the ratio of the number of bonds accepted into the invasion cluster with values "between $x$ and $x+d x$ " by time $n$, to the total number of bonds assigned values in $[x, x+d x]$ by time $n$. The denominator of this quantity thus counts both bonds in the cluster and those on its boundary.

It was observed [4] that, for large times, the acceptance profile approaches a step function which is unity below a certain value of $x$, and zero beyond that value. The important observation is that the cutoff value coincides (within the quoted uncertainties) with the percolation threshold of the graph under study.

2) The geometry of the large-time invaded region appears to be "scale invariant." Thus, at least numerically, it may be assigned a lattice "Hausdorff" (or "fractal") dimension. The values thus obtained [4] agree reasonably well with both those found by direct numerical simulation of the "incipient cluster" of ordinary percolation, and those computed, according to the usual scaling theories, from a knowledge of the gap exponents and correlation length exponents of standard percolation.

3) It has been argued [4] that the rate of convergence of the acceptance profile to a step function contains information on the correlation length exponent, $v$, of ordinary percolation. The exponents thus obtained [4] also agree fairly well with the accepted values.

Further evidence for the assertion that invasion reproduces percolation at threshold has been obtained via an exact solution of the invasion model on the Cayley tree [5]. Here a function different than that introduced in [4], but also called the acceptance profile, was shown to approach a step function with cutoff equal to the percolation threshold of the Cayley tree. The "Hausdorff" dimension of the invaded region was computed in terms of the root mean square cluster radius, reproducing the standard Cayley tree result. Finally, the rate of convergence of the "modified acceptance profile" was used to determine the gap exponent, 4 , which again agreed with the well-known Cayley tree value for ordinary percolation.

\section{Summary of Results}

Our results address various aspects of points 1, 2, and 3 presented above. We begin in Sect. 2 by defining three versions of the invasion model - which are useful for different elements of the subsequent proofs - and showing that the versions are equivalent. The relevant random variables are also introduced in Sect. 2. 
In Sects. 3 and 4, we consider the (infinite-time) behavior of the acceptance profile and a closely related quantity, the empirical distribution function. The latter function, which is perhaps the more useful one for theoretical work, is defined as follows: The empirical distribution at value $x$ and time $n, \mathbf{Q}_{n}(x)$, is $(1 / n)$ times the number of bonds accepted with value less than or equal to $x$. Thus, up to normalization, the empirical distribution is essentially the integral of the acceptance profile. As such, the evidence presented earlier indicates that it should scale linearly with $x$ below the percolation threshold, and approach 1 above threshold. That this (infinite-time) behavior occurs with probability one is proved in Sect. 3, modulo the usual conjectures of rigorous percolation theory.

By the above proviso it is meant that the linear behavior of $\lim _{n \rightarrow \infty} \mathbf{Q}_{n}(x)$ is established for $x$ below the point, $\pi_{c}$, at which the expected cluster size diverges, and that $\lim _{n \rightarrow \infty} \mathbf{Q}_{n}(x)=1$ is proved for $x$ greater than $\bar{p}_{c}$, the half-space percolation threshold. It is worth remarking that in $d=2$, it is known [6-8] that $\pi_{c}=p_{c}=\bar{p}_{c}$. Furthermore, equality of these critical points is widely believed to hold in all dimensions. Lacking the strong result $\pi_{c}=p_{c}=\bar{p}_{c}$ for $d \geqq 3$, we can nonetheless establish a.s. convergence of a subsequence to a linear function for $x<p_{c}$, and that for $x>p_{c}, \mathbf{Q}_{n}(x)$ converges to 1 with nonzero probability.

In order to make contact with the numerical work, in Sect. 4 we define a quantity which we identify as the acceptance profile, $a_{n}(x)$. For finite $n$, it is shown that this quantity is continuous. However, as observed numerically, continuity fails for infinite times. In particular, we prove that

$$
\lim _{n \rightarrow \infty} a_{n}(x)=\left\{\begin{array}{ll}
1 & x<\pi_{c} \\
0 & x>\bar{p}_{c}
\end{array} .\right.
$$

Again, modulo the conjecture $\pi_{c}=\bar{p}_{c}$, this confirms the observations of Wilkinson and Willemsen [4].

In Sect. 5, we examine the geometry of the invaded region. Although we cannot yet establish the existence of a nontrivial lattice "Hausdorff" dimension, we prove the weaker result that, at least in $d=2$, the invaded region has zero volume fraction, with probability one.

To our knowledge, the surface to volume ratio of the invaded region has not been measured numerically. Nevertheless, such a quantity provides additional evidence that the geometry of the invaded region resembles that of percolation configurations at threshold. This follows from the theorem (see [9] and [26]) that, for $p>p_{c}$, the surface to volume ratio of the infinite cluster(s) in ordinary percolation is $(1-p) / p$, and from a related theorem [10] which says that, at threshold, the surface to volume ratio of large finite clusters is asymptotically bounded below by $\left(1-p_{c}\right) / p_{c}$. In Sect. 5 , we prove that with probability one, the (infinite-time) surface to volume ratio of the invaded region is bounded above and below by $\left(1-\pi_{c}\right) / \pi_{c}$ and $\left(1-\bar{p}_{c}\right) / \bar{p}_{c}$, respectively. Assuming the usual conjecture, this is of course the appropriate ratio for percolation at threshold.

In Sect. 6, we examine the rate of convergence of a quantity related to the "modified acceptance profile" introduced in [5]. For our purposes, the modified acceptance profile, $b_{n}(x)$, is taken to be the probability density for acceptance of 
value $x$ at time $n$, so that $B_{n}(y) \equiv \int_{y}^{1} b_{n}(x) d x$ is the probability that the $n^{\text {th }}$ value accepted is greater than $y$. On the Cayley tree, the asymptotic decay rate of $B_{n}(y)$ was determined for both $y=p_{c}$ and $y>p_{c}$ [5]. In the latter case (i.e., $y>p_{c}$ ), it was found that $B_{n}(y)$ vanishes as $e^{-c n}$ as $n \rightarrow \infty$. In Sect. 6, we use the results [11] of rigorous percolation theory to provide the following lower bound on the convergence of $B_{n}$ :

$$
B_{n}(y) \geqq h(y) e^{-c(y) n(d-1) / d} \quad \forall y>p_{c} .
$$

We also supplement the bound (1.2) with an upper bound of the same form

$$
B_{n}(y) \leqq h^{\prime}(y) e^{-c^{\prime}(y) n^{(d-1) / d}} \quad \forall y>1-\pi_{c}^{*},
$$

where $\pi_{c}^{*}$ is the critical point at which the expected cluster size of $(d-1)$-cells diverges. In $d=2$, it is known $[12,13]$ that $1-\pi_{c}^{*}=p_{c}$, so that the asymptotic decay is determined by (1.2) and (1.3). In higher dimensions, $1-\pi_{c}^{*}>p_{c}$; nevertheless, we conjecture that a bound of the form (1.3) holds for all $y>p_{c}$. The Cayley tree result is seen to correspond to the " $d \rightarrow \infty$ limit" of this behavior.

Finally, in the appendix, we provide a proof that in $d=2$, the infinite-time invaded regions which evolve from different (finite) starting sets differ on only a finite number of bonds.

\section{Preliminaries}

The process of invasion percolation has already been described as two equivalent procedures. It turns out that there is a third process which allows, in a direct fashion, the incorporation of well established results from ordinary percolation. Indeed, it is by this method that the majority of the results have been rigorously established. Below we describe all three procedures and demonstrate their equivalence.

\section{Dynamic Growth}

This procedure resembles most closely the numerical simulations. Here we follow, bond by bond, a dynamic evolution of the invaded region. At $\mathbf{t}=0$, random values uniformly distributed between zero and one are assigned to the $2 d$ bonds emanating from the origin. These numbers are compared, and the bond with the smallest value is chosen. (With probability one, the choice is unambiguous.) The chosen bond constitutes the invaded region at time $t=1$. At time $\mathbf{t}=n-1$, values in $[0,1]$ are assigned, at random, to any previously unassigned bonds which have an endpoint in what is currently the invaded region. Then the values that have been assigned to all those bonds which have not yet been chosen are compared and the bond with the smallest value joins the existing cluster.

\section{Deterministic Invasion}

In this approach, the rules of invasion are the same as in 1, except that the random numbers (which are again uniformly distributed in $[0,1]$ ) are assigned to every bond of the lattice before the process begins. Thus the sample space of all 
realizations is $[0,1]^{\mathbb{B}_{d}}$, and the probability measure is the infinite product of Lebesgue measures on the unit interval. In this model it is seen that, once chosen, a $\Gamma \in[0,1]^{\mathbb{B}_{d}}$ determines the entire history of the invasion.

\section{Percolation Cluster Method}

This is a third, intermediate scheme which is dynamic in the sense of 1 , but provides advance, global information as in 2 . We let $y \in(0,1)$ and consider configurations of occupied bonds at density $y$. The set, $\Omega$ of all such configurations (i.e., the set of all subsets of $\mathbb{B}_{d}$ ) is endowed with Bernoulli (product) measure at density $y, \mu_{y}$. The invasion percolation problem is defined by a combination of the rules described in 1 and 2 . First one picks an $\omega \in \Omega$. Then, as in 1 , values are dynamically assigned to the bonds as they join the boundary of the invaded region. Here, however, if a bond is occupied, its value is assigned uniformly in $[0, y]$, while an unoccupied bond is assigned a random value with a uniform distribution in $(y, 1]$. Once values have been assigned to all bonds on the boundary of the existing cluster, the bond with the smallest value is the next one invaded.

Equivalence of the Three Methods. In this paper, we will be concerned with the behavior of the invaded region, as described by certain collections of random variables. These random variables have the same distributions for the three processes. The key observation in this regard is that the finite-time invaded regions are "self-determined clusters," as defined in [14]. For our purposes this means the following:

Let $\mathbf{C} \subset \mathbb{B}^{d}$ be a connected cluster of $n$ bonds containing the origin, and let $\partial \mathbf{C}$ denote its boundary (i.e., $\partial \mathbf{C}$ consists of those bonds not in $\mathbf{C}$ which share a vertex with a bond of $\mathbf{C}$ ). Then the event, $\mathbf{I}_{\mathbf{C}}$, that at time $\mathbf{t}=n$ the invaded region is $\mathbf{C}$ depends only on the values assigned to the bonds of $\mathbf{C}$ and those on the boundary, $\partial \mathbf{C}$. The important point is that $\mathbf{I}_{\mathbf{C}}$ is therefore determined by a relatively small set of variables (e.g., in the dynamic growth model, $\mathbf{I}_{\mathbf{C}}$ may be identified with a subset of $\left.[0,1]^{|\mathbf{C}|+|\partial \mathbf{C}|}\right)$.

Using the above observation, it is easy to establish that $\mathbf{I}_{\mathbf{C}}$ has the same probability in all three models. To this end, consider further the invasion pattern event, $\mathbf{I}_{\mathbf{C} ; \mathbf{P}} \subset \mathbf{I}_{\mathbf{C}}$, that the cluster $\mathbf{C}$ has been invaded through a particular sequence, $\mathbf{P}$, of steps. Let us demonstrate the (stronger) result that the events $\mathbf{I}_{\mathbf{C} ; \mathbf{P}}$, which form a disjoint partition of $\mathbf{I}_{\mathbf{C}}$, have the same probabilities for the three processes. Consider the set of realizations contributing to an $\mathbf{I}_{\mathbf{C} ; \mathbf{P}}$ in the deterministic invasion picture. The invasion pattern, $\mathbf{P}$, allows us to transcribe this set into the (measurable) set of $|\mathbf{C}|+|\partial \mathbf{C}|$ random numbers which, by fiat, "would have" produced the same sequence of events in the dynamic growth model. Evidently, the two $\mathbf{I}_{\mathbf{C} ; \mathbf{P}}$ 's have the same probabilities. A similar argument holds for the percolation cluster method. The only necessary observation is that the $|\mathbf{C}|+|\partial \mathbf{C}|$ bond values in a configuration $\Gamma \in \mathbf{I}_{\mathbf{C} ; \mathbf{p}}$ also determine the pattern of density $y$ occupied bonds in the corresponding realization of the percolation cluster process.

Below we will introduce the random variables which form the basis of our investigation. We need only condition on the appropriate $\mathbf{I}_{\mathbf{C}}$ or $\mathbf{I}_{\mathbf{C} ; \mathbf{P}}$ in order to show that these random variables have the same (conditional) distributions in the 
three languages. As an example, consider the random variable $\mathbf{x}_{n}$ which represents the value of the $n^{\text {th }}$ bond absorbed. Here we simply condition on any $\mathbf{I}_{\mathbf{C} ; \mathbf{P}}$ with $|\mathbf{C}| \geqq n$.

The Random Variables. We begin by defining functions related to the random variables $\mathbf{x}_{n}$, introduced above. Let $y \in[0,1]$ and define

$$
\mathbf{X}_{n}(y)= \begin{cases}1 ; & \text { if } \mathbf{x}_{n} \leqq y \\ 0 ; & \text { otherwise }\end{cases}
$$

The empirical distribution function is given by:

$$
\mathbf{Q}_{n}(y)=\frac{1}{n} \sum_{j=1}^{n} \mathbf{X}_{n}(y) .
$$

Next, we denote by $\mathbf{R}_{n}$ the random number of new bonds which must be checked after selection of the $n^{\text {th }}$ bond (e.g., $\mathbf{R}_{0}=2 d, \mathbf{R}_{1}=2 d-1$, etc.). Evidently, $\forall n \geqq 1,0 \leqq \mathbf{R}_{n} \leqq 2 d-1$. The total number of bonds which have been looked at prior to the selection of the $(n+1)^{\mathrm{st}}$ bond will be denoted by

$$
\mathbf{L}_{n} \equiv \sum_{j=0}^{n} \mathbf{R}_{j}
$$

Observe that $n<\mathbf{L}_{n}=n+$ (no. of bonds which are currently in the boundary of the cluster).

It is often convenient to assign the values to the $\mathbf{R}_{n}$ new bonds in some definite order. This may be implemented via some local rule (e.g., in $d=2$, we can agree to go counterclockwise), or by deterministically ordering the lattice once and for all. Having done so, we may consider the random variable $\mathbf{v}_{k}$ which represents the $k^{\text {th }}$ value checked. We also define

$$
\mathbf{V}_{k}(y)= \begin{cases}1 ; & \text { if } \mathbf{v}_{k} \leqq y \\ 0 ; & \text { otherwise }\end{cases}
$$

The empirical distribution function of values looked at by time $\mathbf{t}=n$ is given by:

$$
\mathbf{P}_{n}=\frac{1}{\mathbf{L}_{n}} \sum_{k=1}^{\mathbf{L}_{n}} \mathbf{V}_{k}(y) \text {. }
$$

As indicated by the following proposition, the large $n$ behavior of $\mathbf{P}_{n}$ is not particularly interesting.

Proposition 2.1. With probability one, $\mathbf{P}_{n}(y) \rightarrow y$.

Proof. This is most easily seen in the context of the dynamic growth picture. Here, although it is clear that the numbers $\mathbf{R}_{n}$ depend on all values previously checked, by definition, the distribution of the $\mathbf{v}_{k}, \mathbf{L}_{n-1}<k \leqq \mathbf{L}_{n}$, does not. The desired result is a consequence of the standard law of large numbers.

Corollary. For every interval $(x, y) \subset[0,1]$, the growth of the empirical distribution function is (asymptotically) bounded as follows:

$$
\lim \sup \left|\mathbf{Q}_{n}(x)-\mathbf{Q}_{n}(y)\right| \leqq(2 d-1)|x-y| \text { with probability } 1 .
$$


Proof. This follows from the above proposition and the observation that the number of values selected in any interval is smaller than the number of values looked at in that interval. Explicitly,

$$
\lim \sup \left|\mathbf{Q}_{n}(x)-\mathbf{Q}_{n}(y)\right| \leqq \lim \sup \frac{\mathbf{L}_{n}}{n}\left|\mathbf{P}_{n}(x)-\mathbf{P}_{n}(y)\right| \leqq(2 d-1)|x-y| .
$$

\section{The Empirical Distribution Function}

In this section, we prove some results concerning the large $n$ behavior of the empirical distribution function, $\mathbf{Q}_{n}(x)$. As explained in the introduction, this quantity is basically the integral of the acceptance profile, studied numerically in [4]. Thus it is expected to have dramatically different behaviors above and below the threshold, $p_{c}$, of the associated Bernoulli percolation model. The two regimes are examined separately.

\section{(i) Invasion Above Threshold}

Here, as in much of what follows, we employ the percolation cluster method. The basic idea is simple: We pick $x>p_{c}$ and draw a Bernoulli configuration of occupied bonds at density $x$. By definition, the occupied bonds form infinite clusters with probability one. Since, if everchecked, an occupied bond will be assigned a value less than or equal to $x$, it is clear that once the invaded region comes into contact with an infinite cluster, it will never again select a value larger than $x$ (see Fig. 1).

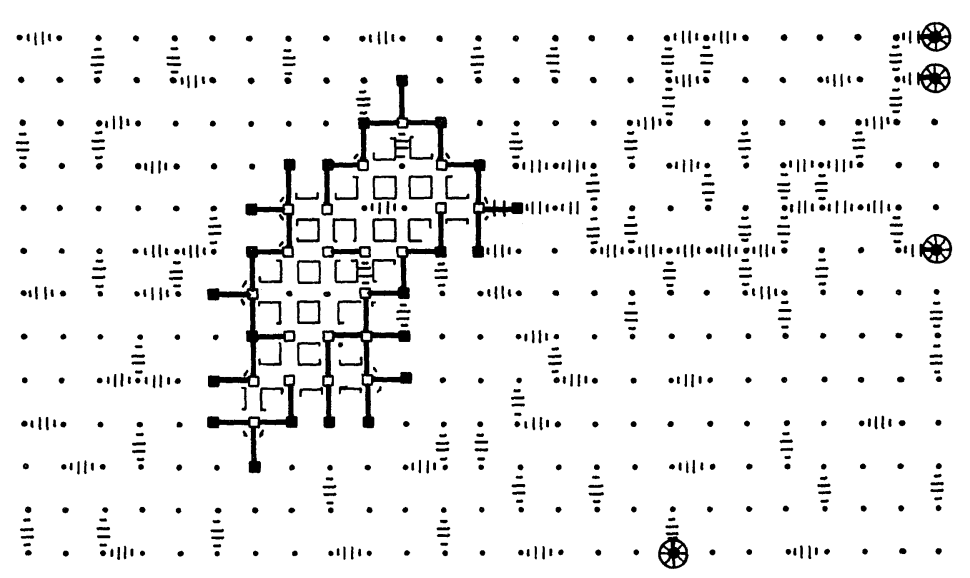

$$
\begin{aligned}
& \because \because \quad \text { Invaded Region } \\
& \because \quad \text { Boundary of Invaded Region } \\
& \because 11 \cdot \text { Occupied Bond } \\
& \text { Indicates Point Connected to Infinity }
\end{aligned}
$$

Fig. 1. Invaded region contacts the infinite cluster 
As an illustration of this method, we prove the following:

Theorem 3.1. Let $x>p_{c}$. Then with nonzero probability,

$$
\mathbf{Q}_{n}(y)=1 \quad \forall n \text { and } \forall y \geqq x .
$$

Proof. Since $x>p_{c}$, the event that we have selected a percolation configuration in which the origin is part of an infinite cluster has nonzero probability.

Under the hypothesis of half-space percolation, it is possible to obtain a far stronger result.

Definition. Let $H_{\infty}(0) \subset \Omega$ denote the set of configurations in which the origin is attached to an infinite cluster of bonds in the half space $\left(x_{1}>0 ;-\infty \leqq x_{j} \leqq+\infty\right.$, $j=2, \ldots, d)$, and define $\mu_{p}\left[H_{\infty}(0)\right] \equiv \bar{P}_{\infty}(p)$. The threshold for half-space percolation is given by

$$
\bar{p}_{c}=\inf \left\{p \in[0,1] \mid \bar{P}_{\infty}(p)>0\right\} .
$$

Remark. Evidently $\bar{p}_{c} \geqq p_{c}$. It is widely believed that $p_{c}=\bar{p}_{c}$ in all dimensions, but as of yet, this has only been rigorously established in $d=2$ [13].

Theorem 3.2. Let $y>\bar{p}_{c}$. Then, with probability one, only finitely many of the $\mathbf{X}_{n}(y)$ are zero.

Proof. Let $\Lambda_{m}$ denote the cube of side $2 m$ centered at the origin

$$
\Lambda_{m}=\left\{x \in \mathbb{Z}^{d}|\forall i=1,2, \ldots, d \quad| x_{i} \mid \leqq m\right\},
$$

and let $\partial \Lambda_{m} \subset \Lambda_{m}$ denote its boundary

$$
\partial \Lambda_{m}=\left\{x \in \Lambda_{m}|\exists i=1,2, \ldots, d \quad| x_{i} \mid=m\right\} .
$$

We define $n_{\Lambda_{m}}$ to be the stopping time which represents the number of bonds that have been absorbed when the invaded region "breaks out" of $\Lambda_{m}$. In other words, $n_{\Lambda_{m}}$ is the earliest time at which a site in $\partial \Lambda_{m}$ becomes attached to the invaded region.

The strategy of the proof is roughly as follows: At each "break-out" time, $n_{\Lambda_{m}}$, the invaded region has a fresh chance to come into contact with an infinite cluster of density $y$ occupied bonds in $\mathbb{Z}^{d} \backslash \Lambda_{m}$. If $y>\bar{p}_{c}$, the probability of this event is bounded away from zero. Moreover, one can construct infinitely many boxes, each providing a new chance. Thus, the invaded region should eventually contact an infinite cluster.

Let $y>\bar{p}_{c}$ and consider percolation configurations at density $y$. Let $\mathbf{s}$ denote the event that at time $\mathbf{t}=n_{\Lambda_{m}}$, the invasion has broken out at the site $s \in \partial \Lambda_{m}$. Observe that the event $\mathbf{s}$ depends only on the distribution of values assigned to those bonds with both endpoints in $\Lambda_{m}$ and at most one endpoint in $\partial \Lambda_{m}$. It is therefore independent of the event that $s$ is attached to infinity by a path of occupied bonds in the complement of the interior of $\Lambda_{m}$. The latter event occurs with probability not smaller than $\bar{P}_{\infty}(y)$.

Now let

$$
\mathbf{M}_{n}(y)=\sum_{j=1}^{n}\left[1-\mathbf{X}_{j}(y)\right]
$$

denote the number of unoccupied bonds which have been invaded at time $n$. Conditioning on the events $\mathbf{s}$, and using the above observation, it is seen that 
$\forall k \leqq\left(\right.$ roo. of bonds in $\left.\Lambda_{m}\right)$,

$$
\operatorname{Prob}\left(\mathbf{M}_{\infty}(y)=\infty \mid \mathbf{M}_{n_{\Lambda_{m}}}(y) \geqq k\right) \leqq 1-\bar{P}_{\infty}(y) .
$$

Letting $\Lambda_{m} \uparrow \mathbb{Z}^{d}$, we obtain

$$
\operatorname{Prob}\left(\mathbf{M}_{\infty}(y)=\infty\right) \leqq \operatorname{Prob}\left(\mathbf{M}_{\infty}(y) \geqq k\right)\left[1-\bar{P}_{\infty}(y)\right] .
$$

Taking the limit $k \rightarrow \infty$, we conclude that

whenever $y>\bar{p}_{c}$.

$$
\operatorname{Prob}\left(\mathbf{M}_{\infty}(y)=\infty\right)=0,
$$

Corollary. $\forall y \geqq \bar{p}_{c}$,

$$
\mathbf{Q}_{n}(y) \rightarrow 1 \text { with probability } 1 .
$$

Proof. This follows immediately if $y>\bar{p}_{c}$. The result for $y=\bar{p}_{c}$ is a consequence of the corollary to Proposition 2.1 .

\section{(ii) Invasion Below Threshold}

We now investigate the behavior of the empirical distribution function for $x<p_{c}$. The results that follow indicate that the distribution is uniform below threshold.

Theorem 3.3. For every $y<p_{c}$, there exists a (random) subsequence $\left(n_{k}\right)$ and $a$ positive (random variable) $C$, bounded away from 0 and $\infty$, such that for every $x<y$,

$$
\mathbf{Q}_{n_{k}}(x) \rightarrow C x \text { with probability } 1 .
$$

Proof. This is again most easily seen in terms of the percolation cluster method. Choose $y<p_{c}$ and let $\omega \in \Omega$. With $\mu_{y}$ probability one, the occupied bonds of $\omega$ form finite clusters. The key observation is that whenever the invaded region contacts one of these clusters, the entire cluster must be absorbed prior to further absorption of unoccupied bonds.

Let $n_{1}, n_{2}, \ldots, n_{k}, \ldots$ denote random variables with values equal to the total number of bonds absorbed immediately after absorption of the $1^{\text {st }}, 2^{\text {nd }}, \ldots, k^{\text {th }}, \ldots$ occupied clusters. Observe that the $n_{k}$ are in fact stopping times. They are characterized by the condition that $\mathbf{t}$ is one of the $n_{k}$ 's iff (1) $\mathbf{x}_{t-1} \leqq y$ and (2) each bond on the boundary of the invaded region at time $\mathbf{t}$ has value greater than $y^{1}$. Note that, by the definition of the stopping times, at any $\mathbf{t}=n_{k}$, all occupied bonds which have been checked have also been absorbed. Thus

which impiies that

$$
\sum_{j=1}^{n_{k}} \mathbf{X}_{j}(y)=\sum_{j=1}^{L_{n_{k}}} \mathbf{V}_{j}(y)
$$

$$
n_{k} \mathbf{Q}_{n_{k}}(y)=\mathbf{L}_{n_{k}} \mathbf{P}_{n_{k}}(y) \text {. }
$$

Let us denote by $s_{k}$ the size of the $k^{\text {th }}$ cluster of occupied bonds which has been absorbed:

$$
S_{k}=\sum_{j=n_{k-1}+1}^{n_{k}} \mathbf{X}_{j}(y) .
$$

1 Although the $n_{k}$ 's depend on the value of $y$ chosen, we shall suppress the $y$-dependence in our notation when no confusion should result. In case of ambiguity, we shall use the notation $n_{k}^{(y)}$ 
In terms of this quantity

so that $\forall x<y$

$$
\mathbf{Q}_{n_{k}}(y)=\frac{1}{n_{k}} \sum_{j=1}^{k} s_{j}
$$

$$
\mathbf{Q}_{n_{k}}(x) / \mathbf{Q}_{n_{k}}(y)=\frac{\sum_{j=1}^{k} s_{j} f_{x}(j)}{\sum_{j=1}^{k} s_{j}} .
$$

In the above, $f_{x}(j)$ is the fraction of the $j^{\text {th }}$ occupied cluster absorbed with value less than $x$.

The event that a given cluster of $\omega$ is invaded is independent of the ultimate distribution of values which are assigned within the cluster. Thus each $f_{x}(j)$ depends only on $s_{j}$ (not on $\omega$ ), and for each value of $s_{j}$ may be calculated according to the usual (i.e., binomal) distribution. It therefore follows from (3.12) and the law of large numbers that

$$
\mathbf{Q}_{n_{k}}(x) / \mathbf{Q}_{n_{k}}(y) \rightarrow x / y \text { with probability } 1 .
$$

The theorem is proved by taking a further subsequence, also denoted by $\left(n_{k}\right)$, which ensures that $\mathbf{Q}_{n_{k}}(y) \rightarrow C y$. That $C$ is bounded away from 0 and $\infty$ is guaranteed by Eq. (3.9) and Proposition 2.1:

$$
y(2 d-1) \geqq \lim _{k \rightarrow \infty} \mathbf{Q}_{n_{k}}(y) \geqq y,
$$

where we have used the obvious bound $(2 d-1)+(2 d / n) \geqq L_{n} / n \geqq 1$.

Remark. By choosing a sequence of stopping times based on a fixed sequence $y_{k} \rightarrow p_{c}$, Theorem 3.3 can be improved to show that there is a single (random) subsequence $\left(n_{k}\right)$, not depending on any $y$, so that for all $x \leqq p_{c}, \mathbf{Q}_{n_{k}}(x) \rightarrow C x$ with probability 1 . In any case, we note that if the two critical points for Bernoulli percolation, $\bar{p}_{c}$ and $\pi_{c}$ [defined in (3.17)] coincide, as they do in $d=2$ and should for any $d$, then it follows from Theorems 3.5 and 5.1 that with probability one, $\mathbf{Q}_{n}(x)$ $\rightarrow\left(1 / p_{c}\right) x$ for all $x \leqq p_{c}$.

Let us define

$$
\mathbf{Q}_{n}^{\prime}(x) \equiv\left(n / L_{n}\right) \mathbf{Q}_{n}(x) .
$$

In terms of this quantity, the above theorem can be restated as: For every $x<p_{c}$, there exists a subsequence $\left(n_{k}\right)$ such that

$$
\mathbf{Q}_{n_{k}}^{\prime}(x) \rightarrow x \text { with probability } 1 \text {. }
$$

The proof of this (perhaps more transparent) reformulation is easily seen, again by using Eq. (3.9) and Proposition 2.1.

Even this reformulation is, nonetheless, somewhat unsatisfactory since we have established convergence only for a subsequence. Indeed, the distribution of values absorbed in the times $n_{k}<\mathbf{t} \leqq n_{k+1}$ - when typically a cluster of occupied bonds is being invaded - could be badly skewed towards lower values. In order to ensure that this skewing does not effect the large $n$ behavior of $\mathbf{Q}_{n}^{\prime}(x)$, we must have some control over the size of the occupied clusters which are encountered by the invaded region. It turns out that finite expected size of connected clusters is a sufficient condition. That is, if $C(0)$ denotes the connected cluster of the origin in the 
Bernoulli system, and $\mathbf{E}_{p}(|C(0)|)$ denotes its expected size, then we consider densities less than

$$
\pi_{c}=\inf \left\{p \in[0,1] \mid \mathbf{E}_{p}(|C(0)|)=\infty\right\} .
$$

We first establish the following property of the stopping times $n_{k}^{(y)}$ when $y<\pi_{c}$.

Lemma 3.4. Let $y<\pi_{c}$, and denote by $n_{k}$ the stopping time corresponding to absorption of the $k^{\text {th }}$ cluster of density y occupied bonds. Then, with probability one,

$$
\lim _{k \rightarrow \infty} \frac{\mathbf{L}_{n_{k+1}}-\mathbf{L}_{n_{k}}}{\mathbf{L}_{n_{k}}}=0 .
$$

Proof. We first show that $\mathbf{E}\left[\left(\mathbf{L}_{n_{k+1}}-\mathbf{L}_{n_{k}}\right)^{2}\right]$ is bounded above uniformly in $k$. To this end, observe that

$\mathbf{L}_{n_{k+1}}-\mathbf{L}_{n_{k}} \leqq\left[\right.$ the size of the $(k+1)^{\text {st }}$ cluster absorbed $]$

$+\left[\right.$ the number of bonds on the boundary of the $(k+1)^{\text {st }}$ cluster absorbed]

$+\left[\right.$ the number of bonds checked from the time $\mathbf{t}=n_{k}$ until the first bond of the $(k+1)^{\text {st }}$ occupied cluster has been contacted].

The first of the above three quantities has already been denoted by $s_{k+1}$. Let us denote the third by $h_{k}$. Since the boundary of the $(k+1)^{\text {st }}$ cluster absorbed cannot exceed $(2 d-1) s_{k+1}$, we have

$$
\mathbf{L}_{n_{k+1}}-\mathbf{L}_{n_{k}} \leqq 2 d s_{k+1}+h_{k} .
$$

From the dynamic growth model, it is clear that $\forall m \geqq 1$

$$
\operatorname{Prob}\left(h_{k} \geqq m\right)=(1-y)^{m-1} .
$$

Thus, if we can show that $\operatorname{Prob}\left(s_{k+1} \geqq m\right)$ also decays exponentially in $m$, uniformly in $k$, then $\mathbf{L}_{n_{k+1}}-\mathbf{L}_{n_{k}}$ has finite moments of all orders.

To this end, consider the first bond of the $(k+1)^{\text {st }}$ occupied cluster to be invaded. (This is the bond which, when checked, determines the value of the random variable $\left.h_{k}\right)$. The event $\left\{s_{k+1} \geqq m\right\}$ is the event that this bond is connected to at least $(m-1)$ other occupied bonds which lie in the complement of the set of bonds already checked. Relaxing this constraint, it follows that $\forall k$, $\operatorname{Prob}\left(s_{k+1} \geqq m\right)$ is bounded above by the probability that the number of occupied bonds attached to a pair of nearest neighbor sites is at least $(m-1)$. For $y<\pi_{c}$, it is known that the latter quantity decays exponentially in $m[15,16]$.

The lemma is now easily established. Let $\varepsilon>0$. Then

$$
\begin{aligned}
\sum_{k=1}^{\infty} \operatorname{Prob}\left[\frac{\mathbf{L}_{n_{k+1}}-\mathbf{L}_{n_{k}}}{\mathbf{L}_{n_{k}}}>\varepsilon\right] & \leqq \sum_{k=1}^{\infty} \operatorname{Prob}\left[\frac{\mathbf{L}_{n_{k+1}}-\mathbf{L}_{n_{k}}}{k}>\varepsilon\right] \\
& \leqq \frac{1}{\varepsilon^{2}} \sum_{k=1}^{\infty} \frac{1}{k^{2}} \mathbf{E}\left[\left(\mathbf{L}_{n_{k+1}}-\mathbf{L}_{n_{k}}\right)^{2}\right]
\end{aligned}
$$


by the Tschebycheff inequality. Since the above quantity is finite, we may apply the Borel-Cantelli lemma to conclude that

$$
\frac{\mathbf{L}_{n_{k+1}}-\mathbf{L}_{n_{k}}}{\mathbf{L}_{n_{k}}} \rightarrow 0 \text { with probability } 1 .
$$

Theorem 3.5. For every $y \leqq \pi_{c}$,

$$
\mathbf{Q}_{n}^{\prime}(y) \rightarrow y \text { with probability } 1 \text {. }
$$

Proof. Let $\Gamma \in[0,1]^{\mathbb{B}_{d}}$ and $y<\pi_{c}$. Denote by $n_{j}(\Gamma), j=1,2, \ldots$, the "stopping times" which correspond to absorption of clusters of bonds with values $\leqq y$ occurring in this realization. Let $m$ be any positive integer and denote by $k(\equiv k(m))$ the largest $k$ such that $n_{k} \leqq m$. Then

$$
\frac{\mathbf{L}_{n_{k+1}}}{\mathbf{L}_{n_{k}}} \mathbf{Q}_{n_{k+1}}^{\prime}(y) \geqq \mathbf{Q}_{m}^{\prime}(y) \geqq \frac{\mathbf{L}_{n_{k}}}{\mathbf{L}_{n_{k+1}}} \mathbf{Q}_{n_{k}}^{\prime}(y) .
$$

By Lemma 3.4, if $y<\pi_{c}$, then $\mathbf{L}_{n_{k}} / \mathbf{L}_{n_{k+1}} \rightarrow 1$ with probability 1 . The result now follows from Eq. (3.16) for $y<\pi_{c}$, and then from the corollary to Proposition 2.1 for $y=\pi_{c}$.

\section{The Acceptance Profile}

In [4], the acceptance profile at value $x$ and time $n$ was defined as the ratio of the number of bonds accepted with values between $x$ and $x+d x$, before time $n$, to the number of bonds checked with values "in that range." Since this quantity, or rather its average over many simulations, is the principal subject of the numerical investigations of invasion percolation, it is worthwhile to translate some of our earlier results into statements about such a function. This is the content of Theorems 4.2 and 4.3 .

However, we must start with a workable definition. This requires some caution since, with nonzero probability, both the numerator and denominator of the above quantity are zero. It turns out that what corresponds best to the object studied is the function

$$
a_{n}(x)=\lim _{\varepsilon \rightarrow 0} \frac{\mathbf{E}\left[\sum_{j=1}^{n}\left[\mathbf{X}_{j}(x+\varepsilon)-\mathbf{X}_{j}(x)\right]\right]}{\mathbf{E}\left[\sum_{k=1}^{\mathbf{L}_{n}}\left[\mathbf{V}_{k}(x+\varepsilon)-\mathbf{V}_{k}(x)\right]\right]} \equiv \lim _{\varepsilon \rightarrow 0} \frac{\mathbf{E}\left[\tilde{\mathbf{Q}}_{n}(x+\varepsilon)-\tilde{\mathbf{Q}}_{n}(x)\right]}{\mathbf{E}\left[\tilde{\mathbf{P}}_{n}(x+\varepsilon)-\tilde{\mathbf{P}}_{n}(x)\right]},
$$

which we will identify as the acceptance profile. In the above, $\widetilde{\mathbf{Q}}_{n}(x)$ and $\widetilde{\mathbf{P}}_{n}(x)$ are the unnormalized analogues of the empirical distributions $\mathbf{Q}_{n}(x)$ and $\mathbf{P}_{n}(x)$. That the definition (4.1) makes sense, and indeed corresponds to a "nice" function, is the content of the following proposition.

Proposition 4.1. For every $n$, the right-hand side of Eq. (4.1) defines an analytic function $a_{n}$.

Proof. By l'Hôpital's rule

$$
a_{n}(x)=\frac{d}{d x} \mathbf{E}\left[\tilde{\mathbf{Q}}_{n}(x)\right] / \frac{d}{d x} \mathbf{E}\left[\tilde{\mathbf{P}}_{n}(x)\right],
$$


provided that everything is well-behaved. That the denominator is well-defined follows from the (not entirely obvious) relation

$$
\mathbf{E}\left[\tilde{\mathbf{P}}_{n}(x)\right]=x \mathbf{E}\left[\mathbf{L}_{n}\right] .
$$

To see this, write $\mathbf{E}\left[\mathbf{L}_{n}\right]=\sum_{j} \mathbf{E}\left[\mathbf{R}_{j}\right]$. The claim is clearly true for $n=0$ (i.e., $\mathbf{E}\left[\tilde{\mathbf{P}}_{0}(x)\right]$ $\left.=2 d x=x \mathbf{E}\left[\mathbf{L}_{0}\right]\right)$. Suppose the result has been established for some $n_{0}$. Then

$$
\mathbf{E}\left[\tilde{\mathbf{P}}_{n_{0}+1}(x)\right]=\mathbf{E}\left[\tilde{\mathbf{P}}_{n_{0}}(x)\right]+\mathbf{E}\left[\tilde{\mathbf{P}}_{n_{0}+1}(x)-\tilde{\mathbf{P}}_{n_{0}}(x)\right] .
$$

The second term may be rewritten as $\left[\sum_{k}(x k) \operatorname{Prob}\left(\mathbf{R}_{n_{0}+1}=k\right)\right]=x \mathbf{E}\left[\mathbf{R}_{n_{0}+1}\right]$ since, regardless of the value of $\mathbf{R}_{n_{0}+1}$, the distribution of values on the new bonds looked at will be uniform. The claim (4.3) now follows by combining this observation with the inductive hypothesis and the expression for $\mathbf{E}\left[\mathbf{L}_{n}\right]$ in terms of the $\mathbf{E}\left[\mathbf{R}_{j}\right]$.

Since $\mathbf{E}\left[\widetilde{\mathbf{Q}}_{n}(x)\right]$ is monotone, it is a.e. differentiable. Furthermore,

$$
\left|\tilde{\mathbf{Q}}_{n}(x+\varepsilon)-\tilde{\mathbf{Q}}_{n}(x)\right| \leqq\left|\tilde{\mathbf{P}}_{n}(x+\varepsilon)-\tilde{\mathbf{P}}_{n}(x)\right|,
$$

so the derivative is bounded. Below we will show that $\mathbf{E}\left[\tilde{\mathbf{Q}}_{n}(x)\right]$ is in fact analytic, and indeed a polynomial.

To do this, we first note that

$$
\mathbf{E}\left[\widetilde{\mathbf{Q}}_{n}(x)\right]=\sum_{j=1}^{n} \operatorname{Prob}\left(\mathbf{x}_{j} \leqq x\right) .
$$

Let $\Lambda_{j}$ denote the cube of side $2 j$ centered at the origin, let $\mathbf{y}_{b}$ denote the uniformly distributed random variable assigned to the bond $b$ in the deterministic invasion procedure and let $W$ denote the ordering of the $\mathbf{y}_{b}$ 's inside $\Lambda_{j}$. The bond which is invaded at time $j$ is a deterministic function, $g(W)$, of the order $W$ but does not otherwise depend on the $\mathbf{y}_{b}$ 's. Thus $\mathbf{x}_{j}=\mathbf{y}_{g(W)}$. Conditioning on the possible orderings, $w$, yields

$$
\operatorname{Prob}\left(\mathbf{x}_{j} \leqq x\right)=\sum_{w} \operatorname{Prob}(W=w) \operatorname{Prob}\left(\mathbf{y}_{g(W)} \leqq x \mid W=w\right) .
$$

For each $j$ and $w$ we number the bonds in increasing order according to $W$, so that

$$
\operatorname{Prob}\left(\mathbf{y}_{g(W)} \leqq x \mid W=w\right)=\operatorname{Prob}\left(U_{k} \leqq x \mid U_{1}<U_{2}<\ldots<U_{m}\right),
$$

where $k$ is the number (in the above ordering) of $g(w), m$ is the number of bonds in $\Lambda_{j}$, and $U_{1}, \ldots, U_{m}$ are independent and uniformly distributed on $[0,1]$. The existence and analyticity of $\frac{d}{d x} \mathbf{E}\left[\tilde{\mathbf{Q}}_{n}(x)\right]$ now follow by combining (4.6), (4.7), (4.8) and the easily verified fact that

$$
\frac{d}{d x} \operatorname{Prob}\left(U_{k} \leqq x \mid U_{1}<U_{2}<\ldots<U_{m}\right)=\text { const } x^{k-1}(1-x)^{m-k} .
$$

Remark. It is worthwhile to note that the analyticity of (4.9), which proves the analyticity of $a_{n}(x)$, deteriorates as $m$ and $k$ increase. E.g., as $m \rightarrow \infty$ and $k / m$ $\rightarrow \lambda \in[0,1],(4.9)$ tends to a delta function at $\lambda$. This is not an artifact of our proof. Indeed, it has been observed numerically that the acceptance profile approaches a 
step function as $n \rightarrow \infty$. Below we will study the limiting behavior of the acceptance profile for all noncritical values of $x$ (i.e., $x \in[0,1] \backslash\left[\pi_{c}, \bar{p}_{c}\right]$ ), and rigorously establish the results indicated by the numerical simulations [4].

Our analysis is facilitated by fixing a concrete realization of the percolation cluster process. To this end, we deterministically order the lattice (with, say, spiral order) thus specifying, for each $n$, the order in which values are assigned to the $\mathbf{R}_{n}$ new bonds. Then the sample space may be realized as $\Omega \otimes \mathbf{A} \otimes \mathbf{B}$, where $\Omega$ is the set of all subsets of $\mathbb{B}^{d}, \mathbf{A}$ is the set of all real sequences with range $[0, x]$ and $\mathbf{B}$ is the set of sequences in $(x, 1]$. The probability measure is just Bernoulli percolation measure at density $x$, crossed with the two infinite products of (normalized) Lebesgue measures. A configuration, $\omega_{\alpha \beta} \equiv((\omega, \alpha, \beta) \mid \omega \in \Omega, \alpha \in \mathbf{A}, \beta \in \mathbf{B})$, determines the invasion uniquely by assigning the value $\alpha_{i}$ to the $i^{\text {th }}$ occupied bond and $\beta_{j}$ to be $j^{\text {th }}$ unoccupied bond examined.

We now show:

Theorem 4.2. For every $x>\bar{p}_{c}$,

$$
\lim _{n \rightarrow \infty} a_{n}(x)=0 .
$$

Proof. Let $x>\bar{p}_{c}$, so that for a.e. $\omega_{\alpha \beta}$, the occupied bonds percolate. Denote by $S_{N}$ the event that by time $\mathbf{t}=N$ the invaded region has already made contact with an infinite cluster. Note that for each $N, S_{N}$ depends on essentially all coordinates of $\omega$, but only on a small number of coordinates of $\alpha$ and $\beta$.

For $n_{1}>n_{2}$, let us define the function

$$
\tilde{\mathbf{P}}_{n_{1} \backslash n_{2}}(x) \equiv \tilde{\mathbf{P}}_{n_{1}}(x)-\tilde{\mathbf{P}}_{n_{2}}(x) .
$$

We first show that $\forall n>N$,

$$
\mathbf{E}\left[\tilde{\mathbf{P}}_{n \backslash N}(x+\varepsilon)-\tilde{\mathbf{P}}_{n \backslash N}(x) \mid S_{N}^{c}\right] \leqq \varepsilon(2 d-1)(n-N) /(1-x),
$$

where $S_{N}^{c}$ is the event that the invaded region has not yet contacted an infinite cluster by time $\mathbf{t}=N$. The relation (4.11) is seen by observing that the integrand is determined by the values, $\beta_{i}$, of the bonds for which $\mathbf{L}_{N}-\tilde{\mathbf{P}}_{N}(x) \leqq i \leqq \mathbf{L}_{n}-\tilde{\mathbf{P}}_{n}(x)$. These are independent of the conditioning since the only $\beta_{i}$ 's on which the event $S_{N}^{c}$ depends are those with $i<\mathbf{L}_{N}-\widetilde{\mathbf{P}}_{N}(x)$. Now the number of $\beta_{i}$ 's such that $\mathbf{L}_{N}-\tilde{\mathbf{P}}_{N}(x)<i \leqq \mathbf{L}_{n}-\tilde{\mathbf{P}}_{n}(x)$ is bounded above by the maximum number of bonds which could have been looked at during times $N<\mathbf{t} \leqq n$. This is simply $(2 d-1)(n-N)$. The integrand counts only those bonds for which $\beta_{i} \in(x, x+\varepsilon]$; hence the factor $\varepsilon /(1-x)$.

The proof is now straightforward. Choose $N>1$ and let $n>N$. Using the usual notation for the indicator function, and the simplified notation $\mathbb{Q}_{n} \equiv \tilde{\mathbf{Q}}_{n}(x+\varepsilon)$ $-\tilde{\mathbf{Q}}_{n}(x)$ and $\mathbb{P}_{n} \equiv \tilde{\mathbf{P}}_{n}(x+\varepsilon)-\tilde{\mathbf{P}}_{n}(x)$ for increments of the empirical distributions, we have

$$
\begin{aligned}
\mathbb{Q}_{n}=\mathbb{Q}_{n}\left[\mathbb{1}_{S_{N}}+\left(1-\mathbb{1}_{S_{N}}\right)\right] & =\mathbb{Q}_{N}+\left[1-\mathbb{1}_{S_{N}}\right] \mathbb{Q}_{n \backslash N} \\
& \leqq \mathbb{P}_{N}+\left[1-\mathbb{1}_{S_{N}}\right]\left[\mathbb{Q}_{N}-\mathbb{P}_{N}\right]+\left[1-\mathbb{1}_{S_{N}}\right] \mathbb{Q}_{n \backslash N} \\
& =\mathbb{P}_{N}+\left[1-\mathbb{1}_{S_{N}}\right]\left[\mathbb{Q}_{n}-\mathbb{P}_{N}\right] \leqq \mathbb{P}_{N}+\left[1-\mathbb{1}_{S_{N}}\right] \mathbb{P}_{n \backslash N}
\end{aligned}
$$


Taking expectations of both sides of the above equation, and using (4.2), (4.3) and the bound (4.11), we obtain

$$
a_{n}(x) \leqq\left[\mathbf{E}\left[\mathbf{L}_{N}\right]+\operatorname{Prob}\left(S_{N}^{c}\right)(2 d-1)(n-N) /(1-x)\right] / \mathbf{E}\left[\mathbf{L}_{n}\right]
$$

so that

$$
\lim \sup a_{n}(x) \leqq \operatorname{Prob}\left(S_{N}^{c}\right)(2 d-1) /(1-x)
$$

Finally recall that, by Theorem $3.2, \operatorname{Prob}\left(S_{N}^{c}\right) \rightarrow 0$ as $N \rightarrow \infty$, which establishes the desired result.

Next, we examine the behavior of the acceptance profile below threshold.

Theorem 4.3. For every $x<\pi_{c}$,

$$
\lim _{n \rightarrow \infty} a_{n}(x)=1 .
$$

Proof. Let us write $a_{n}(x)$ in the form

$$
\begin{aligned}
a_{n}(x) & =1-\lim _{\varepsilon \rightarrow 0}\left(\varepsilon \mathbf{E}\left[\mathbf{L}_{n}\right]\right)^{-1} \mathbf{E}\left[\left(\tilde{\mathbf{P}}_{n}(x+\varepsilon)-\tilde{\mathbf{P}}_{n}(x)\right)-\left(\tilde{\mathbf{Q}}_{n}(x+\varepsilon)-\tilde{\mathbf{Q}}_{n}(x)\right)\right] \\
& \geqq 1-\lim _{\varepsilon \rightarrow 0}(\varepsilon n)^{-1} \mathbf{E}\left[\left(\tilde{\mathbf{P}}_{n}(x+\varepsilon)-\tilde{\mathbf{P}}_{n}(x)\right)-\left(\tilde{\mathbf{Q}}_{n}(x+\varepsilon)-\tilde{\mathbf{Q}}_{n}(x)\right)\right] .
\end{aligned}
$$

It suffices to demonstrate that the limit on the right-hand side of (4.15) tends to zero in the large $n$ limit.

To this end, recall the definition of the stopping times given in Sect. 3.ii. By the reasoning of Theorem 3.3, it is seen that if $n^{*}$ is a $y$ density stopping time, with $y<\pi_{c}$, then

$$
\tilde{\mathbf{Q}}_{n^{*}}(x+\varepsilon)-\tilde{\mathbf{Q}}_{n^{*}}(x)=\tilde{\mathbf{P}}_{n^{*}}(x+\varepsilon)-\tilde{\mathbf{P}}_{n^{*}}(x)
$$

$\forall x, \varepsilon$ such that $x+\varepsilon<y$.

The next observation is somewhat more subtle: If $n^{*}$ is a stopping time in a given realization $\omega_{\alpha \beta}$, then the value of the next stopping time is independent of the values of the $\alpha_{i} \in[0, y]$ for all $i>\tilde{\mathbf{P}}_{n^{*}}(y)$. To see this, recall that at time $\mathbf{t}=n^{*}$, the final bond of the most recently attacked occupied cluster has just been absorbed. Every bond on the boundary of the invaded region is now "unoccupied." The next stopping time will occur when the invaded region contacts and absorbs another occupied cluster. Which cluster it absorbs and how long it takes to get there depend only on what clusters are "nearby" the currently invaded region and on the evolution of the invasion pattern through the intervening unoccupied territory. These quantities are determined exclusively by $\omega$, the values on the current waiting list and the values of $\beta_{j} \in(y, 1], j>\mathbf{L}_{n^{*}}-\tilde{\mathbf{P}}_{n^{*}}(y)$. Hence they are independent of $\alpha_{i}$, $i>\tilde{\mathbf{P}}_{n^{*}}$. The latter values influence only the nature of the invasion pattern after the next occupied cluster has been struck. (Of course, the values of subsequent stopping times will depend on these $\alpha_{i}$.)

Now assume that $n$ is not a stopping time, and let $n^{*}<n$ be the last stopping time before $n$. Conditioned on $n^{*}$, it is obvious that $\tilde{\mathbf{P}}_{n \backslash n^{*}}(x+\varepsilon)-\tilde{\mathbf{P}}_{n \backslash n^{*}}(x)$ is zero unless the invaded region has already made contact with the next occupied 
cluster. ${ }^{2}$ Calling $\sigma_{T}(n)$ the event that at time $n$, the invaded region has spent $T$ units of time in contact with the "next" cluster, it is clear that

$\mathbf{E}\left[\tilde{\mathbf{P}}_{n \backslash n^{*}}(x+\varepsilon)-\tilde{P}_{n \backslash n^{*}}(x) \mid n^{*}\right.$ last stopping time before $\left.n ; \sigma_{T}(n)\right] \leqq \varepsilon(2 d-1) T / x$.

Summing over all values of $T$ and $n^{*}$ (here we conveniently define $\mathbf{t}=0$ to be a stopping time) we have, from (4.16) and (4.17),

$$
[\varepsilon n]^{-1} \mathbf{E}\left[\left(\tilde{\mathbf{P}}_{n}(x+\varepsilon)-\tilde{\mathbf{P}}_{n}(x)\right)-\left(\tilde{\mathbf{Q}}_{n}(x+\varepsilon)-\tilde{\mathbf{Q}}_{n}(x)\right)\right] \leqq \frac{2 d-1}{x} \frac{1}{n} \sum_{T=0}^{n} T \operatorname{Prob}\left[\sigma_{T}(n)\right] .
$$

Now Prob $\left[\sigma_{T}(n)\right]$ is just the probability that at time $\mathbf{t}=n$ the invaded region has spent $T$ units of time engrossed with its most recent cluster. As was shown in Lemma 3.4, this probability tends to zero exponentially in $T$, which establishes the desired result.

\section{Geometry of the Invaded Region}

The results of the previous sections suggest that the configurations generated by invasion percolation may resemble those of ordinary percolation at threshold. Indeed there is much indirect numerical evidence to support such a claim [4]. Here we study the geometry of the invaded region in an attempt to compare it to the (admittedly scarce) rigorous knowledge on the structure of percolation configurations at threshold.

\section{The Surface to Volume Ratio}

Prior to the selection of the $(n+1)^{\text {st }}$ bond, the boundary of the invaded region consists precisely of those bonds which have been checked, but not selected (i.e., the current "waiting list"). The number of such bonds at time $\mathbf{t}=n$ is therefore $\mathbf{L}_{n}-n$, and the surface area to volume ratio is $\left(\mathbf{L}_{n}-n\right) / n$.

One can define a similar notion of boundary for the infinite cluster(s) in ordinary Bernoulli bond percolation at density $p>p_{c}$ : Simply consider all those bonds which are not occupied, but which share an endpoint with a bond of an infinite cluster. It has been shown (see [9] and [26]) that the surface to volume ratio for the infinite cluster(s) in ordinary percolation at density $p>p_{c}$ is precisely $(1-p) / p$.

Below we study the large $n$ limit of $\left(\mathbf{L}_{n}-n\right) / n$, and establish (modulo the usual conjectures of percolation theory) that this reproduces the anticipated surface to volume ratio of ordinary percolation at threshold.

Theorem 5.1. With probability one, the large $n$ limit of the surface to volume ratio has the bounds

$$
\frac{1-\bar{p}_{c}}{\bar{p}_{c}} \leqq \liminf _{n \rightarrow \infty} \frac{\mathbf{L}_{n}-n}{n} \leqq \limsup _{n \rightarrow \infty} \frac{\mathbf{L}_{n}-n}{n} \leqq \frac{1-\pi_{c}}{\pi_{c}}
$$

2 The authors would like to thank the referee for pointing out this simplification 
Proof. By Theorem 3.5, with probability one,

Thus

$$
\frac{n}{\mathbf{L}_{n}} \geqq \frac{1}{\mathbf{L}_{n}} \sum_{j=1}^{n} \mathbf{X}_{n}\left(\pi_{c}\right) \equiv \mathbf{Q}_{n}^{\prime}\left(\pi_{c}\right) \rightarrow \pi_{c}
$$

$$
\limsup _{n \rightarrow \infty} \frac{\mathbf{L}_{n}-n}{n} \leqq \frac{1-\pi_{c}}{\pi_{c}} \text { with probability } 1 \text {. }
$$

On the other hand,

$$
\frac{n}{\mathbf{L}_{n}}=\frac{1}{\mathbf{L}_{n}} \sum_{j=1}^{n} \mathbf{X}_{j}\left(\bar{p}_{c}\right)+\frac{1}{\mathbf{L}_{n}} \sum_{j=1}^{n}\left[1-\mathbf{X}_{j}\left(\bar{p}_{c}\right)\right] \leqq \frac{1}{\mathbf{L}_{n}} \sum_{j=1}^{\mathbf{L}_{n}} \mathbf{V}_{j}\left(\bar{p}_{c}\right)+\left[1-\mathbf{Q}_{n}\left(\bar{p}_{c}\right)\right]
$$

By Proposition 2.1, the first term above converges a.s. to $\bar{p}_{c}$. By the corollary to Theorem 3.2, as $n \rightarrow \infty$, the second term a.s. vanishes. This gives

$$
\liminf _{n \rightarrow \infty} \frac{\mathbf{L}_{n}-n}{n} \geqq \frac{1-\bar{p}_{c}}{\bar{p}_{c}} \text { with probability } 1 \text {. }
$$

Remarks. 1) It follows immediately that if $\pi_{c}=p_{c}=\bar{p}_{c}$, then with probability one, the limit of $\left[\mathbf{L}_{n}-n\right] / n$ exists. That $\pi_{c}=p_{c}=\bar{p}_{c}$ is of course known for $d=2[6,13]$, and is widely believed to hold in all dimensions.

Conversely, should it be the case that with probability 1 a limit exists, the subsequence argument of Theorem 3.3 establishes that the limit is bounded above by $\left(1-p_{c}\right) / p_{c}$. Finally, if the limit exists and is a.s. a constant, it is equal to $\left(1-p_{c}\right) / p_{c}$, since by Theorem $3.1, \forall \varepsilon>0$, the limit must be larger than $\left(1-p_{c}\right) / p_{c}-\varepsilon$ on a set of nonzero measure.

2) The asymptotic surface to volume ratio treated in Theorem 5.1 is defined dynamically. An alternate static definition, analogous to the one used for infinite clusters in [9], is to begin with the infinite-time invaded region $\mathbf{C}$ and its boundary $\partial \mathbf{C}$, intersect these with a cube $\Lambda_{m}$ of side $2 m$ centered at the origin and take the limit of the ratio of the sizes, $\lim \left(\left|\partial \mathbf{C} \cap \Lambda_{m}\right| /\left|\mathbf{C} \cap \Lambda_{m}\right|\right)$, as $m \rightarrow \infty$. It should be possible to show (at least in $d=2$ ) that this definition is equivalent to the one used in Theorem 5.1.

\section{The Volume Fraction}

The above theorem shows that, in some sense, the invaded regions are "all surface." However, in light of the analogous results for positive density infinite clusters mentioned previously [9], this alone does not indicate that the invaded region has a "fractal" or even "scale invariant" structure.

The existence (and value of) a "Hausdorff" dimension can, at this point, only be studied numerically. In lieu of such strong results, it is at least possible to establish - modulo the usual conjectures - that the invaded region has a zero volume fraction. This provides further justification for the assertion that invasion percolation is representative of critical point phenomena.

Theorem 5.2. Let $b \in \mathbb{B}_{d}$ and denote $b y \Theta_{b}$ the event that $b$ is eventually invaded. Then the volume fraction, defined by

$$
\mathscr{V} \equiv \limsup _{m \rightarrow \infty} \frac{1}{\left|\Lambda_{m}\right|} \sum_{b \in \Lambda_{m}} \mathbb{1}_{\Theta_{b}},
$$


satisfies

$$
\mathscr{V} \leqq P_{\infty}\left(\bar{p}_{c}\right) \text { with probability } 1 .
$$

Remark. The percolation probability, $P_{\infty}$, is known to be continuous in $d=2$ [7], so that $P_{\infty}\left(\bar{p}_{c}\right)=0$ and, by Theorem 5.2, $\mathscr{V}=0$. Moreover, continuity is expected, on general grounds, for all finite-range percolation models defined on periodic graphs, so that $\mathscr{V}=0$ should follow in these cases also. In contrast, for a certain class of long-range models in one dimension, it has recently been established [17] that $P_{\infty}$ at threshold is positive (Thouless effect). Indeed, an invasion percolation study on the above type of system may yield interesting and novel results. To construct the relevant invasion processes, follow the procedures of Sect. 2 except assign to each pair of vertices $\{i, j\}$ a random number $[$ in $(0, \infty)]$ with an exponential distribution of parameter $J_{i j}$. The resulting invasion model is then related to the critical behavior of the bond percolation model with

$$
\text { Prob(occupied bond between } i \text { and } j)=1-e^{-\beta J_{i j}}
$$

at its critical value $\beta=\beta_{c}$. These procedures are well-defined provided that $\sum_{j} J_{i j}<\infty$ for every $i$.

Proof of Theorem 5.2. This is essentially a corollary to Theorem 3.1. Let $y>\bar{p}_{c}$ and draw, in the percolation cluster method, a sample of density $y$ occupied bonds. Then, with probability one, except for finitely many bonds, $\mathbb{1}_{\Theta_{b}} \leqq \mathbb{1}_{\mathbf{C}_{b}}$, where $\mathbf{C}_{b}$ is the event that $b$ is part of an infinite cluster. The bound now follows from an application of the Birkhoff ergodic theorem and the continuity from the right of $P_{\infty}(p)[12]$.

\section{Large-Time Asymptotics}

According to Theorem 3.2, $\mathbf{E}\left(1-\mathbf{X}_{n}(y)\right) \rightarrow 0$ if $y>\bar{p}_{c}$ (and, we expect, if $y>p_{c}$ ). Here we examine the rate of this convergence. Indeed, this question is of interest for both $y=p_{c}$ and $y>p_{c}$, with different rates of convergence anticipated for the two cases. Although we address the $y=p_{c}$ question briefly at the end of the section, our principal results concern $y>p_{c}$.

In order to facilitate the discussion, let us introduce the notation

$$
\mathbf{Z}_{n}(y) \equiv 1-\mathbf{X}_{n}(y)
$$

and

$$
B_{n}(y) \equiv \mathbf{E}\left[\mathbf{Z}_{n}(y)\right] .
$$

Thus $B_{n}(y)$ represents the probability that a value greater than $y$ is accepted on the $n^{\text {th }}$ step. We turn attention to the large $n$ asymptotics.

First, however, let us relate $B_{n}(y)$ to other quantities considered in the study of invasion percolation. Observe that

$$
b_{n}(x) \equiv-\frac{d}{d x} B_{n}(x)
$$


has the interpretation of a probability density for acceptance of value $x$ at time $n$. In [5], the quantity $b_{n}(x)$ was computed exactly on the Cayley tree. Although $b_{n}(x)$ was called the acceptance profile in [5], it is related to the acceptance profile of [4] and Sect. 4 by

$$
a_{n}(x)=\frac{1}{\mathbf{E}\left[\mathbf{L}_{n}\right]} \sum_{j=1}^{n} b_{j}(x),
$$

which follows from (4.2), (4.3), and (4.6).

On the Cayley tree, it was found [5] that $\forall y>p_{c}, B_{n}(y)$ vanishes exponentially in $n$. Because of this result, there has been a temptation to conjecture that exponential decay is true in general. Such a conjecture is in fact wrong, as demonstrated by the theorem below. The subexponential decay seems to be a manifestation of the essential singularity [10] in the generating function for percolation clusters.

Theorem 6.1. For every $y>p_{c}$, there is a constant $c(y)<\infty$ such that

with $h(y)>0$.

$$
B_{n}(y) \geqq h(y) e^{-c(y) n^{(d-1) / d}}
$$

Proof. This result follows immediately from Theorem 1 of Aizenman, Delyon and Soulliard [11].

To see this, we use the percolation cluster method. Let $y>p_{c}$ and consider configurations of density $y$ occupied bonds. Now note that if the origin is in a finite cluster of exactly $n-1$ occupied bonds, it must be the case that the $n^{\text {th }}$ bond chosen has value greater than $y$. This implies that

$$
B_{n}(y) \geqq P_{n-1}(y),
$$

where $P_{m}(y)$ is the Bernoulli probability, at density $y$, that the connected component of the origin contains exactly $m$ bonds. Theorem 1 of [11] gives lower bounds on $P_{m}(y)$ of the desired form. It is worth noting that [11] also provides explicit bounds on the constant $c(y)$, in terms of $P_{\infty}(y)$ and $d$.

Although the lower bound of Theorem 6.1 proves that the convergence is subexponential, it leaves open the possibility of a much slower rate of convergence. We believe, however, that the lower bound is precisely the correct asymptotic behavior whenever $y>p_{c}$. Unfortunately, we can only prove this for $d=2$. For general dimension, although we can obtain an upper bound of the form (6.5), the bound is legitimate only for $y$ larger than some value, defined below, which exceeds $p_{c}$ in $d \geqq 3$.

Consider the $y$ density Bernoulli bond system on the lattice $\mathbb{B}_{d}$. By duality, the $(d-1)$-cells (e.g., bonds in $d=2$, plaquettes in $d=3$, etc.) may be regarded as occupied with probability $q=1-y$. If we denote by $\left|C^{*}(0)\right|$ the size of the connected cluster of occupied $(d-1)$-cells of the origin, we may define the critical point

$$
\pi_{c}^{*} \equiv \inf \left\{q \in[0,1] \mid \mathbf{E}_{q}\left(\left|C^{*}(0)\right|\right)=\infty\right\} .
$$

That is, $\pi_{c}^{*}$ is the point at which the expected size of $(d-1)$-cell clusters diverges.

The point $\pi_{c}^{*}$ should not be confused with other critical points of the $(d-1)$-cell system. For example, in $d=3$ there is another transition point, presumably $1-p_{c}$, above which plaquettes form infinite "sheets," as defined and analyzed in [18]. It is 
elementary to show that, for the bond-plaquette system, this transition occurs at a point strictly above $\pi_{c}^{*}$. A closely related, but more subtle result for the site system on $\mathbb{Z}^{3}$ has been established by Campanino and Russo [19].

In Theorem 6.2, an upper bound of the form (6.5) is proved for $y>1-\pi_{c}^{*}$. This establishes the asymptotic behavior $B_{n} \sim \exp \left(-c n^{1 / 2}\right)$ for all $y>p_{c}$ in $d=2$, since it is known $[12,13]$ that $1-\pi_{c}^{*}=p_{c}$ for the two-dimensional system.

Before proving Theorem 6.2 , it is worth noting that the difficulties in extending the higher dimensional result from $1-\pi_{c}^{*}$ to $p_{c}$ are related, but not equivalent to the problems in establishing upper bounds on $P_{n}(y)$ in ordinary percolation. As exploited in the proof of Theorem 6.1, the $P_{n}$ provide a lower bound on the $B_{n}$. Thus, if the results of Theorem 6.2 could be extended, this would also extend the results of Kunz and Soulliard [10] on the asymptotics of the $P_{n}$. The converse is, however, not the case since the $n^{\text {th }}$ bond chosen may be greater than $y$ even if, in the percolation cluster method, the origin is part of a cluster of size much smaller than $n$.

Theorem 6.2. For every $y>1-\pi_{c}^{*}$, there is a constant $c^{\prime}(y)>0$ such that

$$
B_{n}(y) \leqq h^{\prime}(y) e^{-c^{\prime}(y) n^{(d-1) / d}}
$$

with $h^{\prime}(y)<\infty$.

Proof. Again, we use the percolation cluster method. Let $y>1-\pi_{c}^{*}$ and consider the Bernoulli system of density $y$ occupied bonds and density $1-y$ occupied $(d-1)$-cells.

Observe that if $\mathbf{Z}_{n}(y)=1$, then there must be a closed surface of occupied $(d-1)$-cells which surrounds the origin, encloses a volume at least as large as $n$, and has at least one of the $(d-1)$-cells in this surface within a distance $n$ of the origin. However, if it encloses a volume at least as large as $n$, then its surface area must be at least $\kappa(d) n^{(d-1) / d}$, with some explicitly computable $\kappa(d)>0$. Thus $\mathbf{Z}_{n}(y)=1$ implies that within a distance $n$ of the origin, there is a $(d-1)$-cell with connected component of size at least $\kappa(d) n^{(d-1) / d}$. We have

$$
\begin{aligned}
B_{n}(y) & \leqq \operatorname{Prob}_{y}\left[\bigcup_{i:|i| \leqq n}\left\{\left|C^{*}(i)\right| \geqq \kappa(d) n^{(d-1) / d}\right\}\right] \\
& \leqq O\left(n^{d}\right) \operatorname{Prob}_{y}\left[\left\{\left|C^{*}(0)\right| \geqq \kappa(d) n^{(d-1) / d}\right\}\right],
\end{aligned}
$$

where the inequality is a consequence of subadditivity and translation invariance.

It is known $[15,16]$ that for $\pi_{c}^{*}>1-y$, the above probability decays exponentially in $\kappa(d) n^{(d-1) / d}$, with some characteristic rate $\lambda(y)>0$. Thus

$$
B_{n}(y) \leqq 0\left(n^{d}\right) e^{-\kappa \lambda(y) n n^{(d-1) / d}} \leqq h^{\prime}(y) e^{-c^{\prime}(y) n^{(d-1) / d}}
$$

for some $c^{\prime}(y)>0$ and $h^{\prime}(y)<\infty$.

Let us briefly consider the decay of $B_{n}(y)$ for $y=p_{c}$. Nickel and Wilkinson [5] (see also [4]) argue that $B_{n}\left(p_{c}\right) \sim n^{-1 / 4}$, where $\Delta$ is the gap exponent. On the Cayley tree, they find $B_{n}\left(p_{c}\right) \sim n^{-1 / 2}$, consistent with the mean field value for $\Delta$. We have nothing as strong as a mean field bound. We do, however, note that as a consequence of the bound (6.6),

$$
\sum n B_{n}\left(\pi_{c}\right) \geqq \sum n P_{n-1}\left(\pi_{c}\right)=\mathbf{E}_{\pi_{c}}[|C(0)|]=\infty,
$$


so that if $B_{n}\left(\pi_{c}\right) \sim n^{-\tau}$, then $\tau \leqq 2$. In two dimensions, this bound can be strengthened by using the result of van den Berg and Kesten [20]. They provided an explicit bound at threshold for the quantity $K_{n}=\sum_{N \geqq n} P_{N}$ :

$$
K_{n}\left(p_{c}\right) \geqq(1 / 2) n^{-1 / 2} \text { in } d=2 .
$$

From this it follows that there exists a positive constant such that infinitely many of the $P_{n}$ 's have the bound $P_{n}\left(p_{c}\right) \geqq($ const $) n^{-3 / 2}$. Thus by (6.6), if $B_{n}\left(p_{c}\right) \sim n^{-\tau^{\prime}}$ then $\tau^{\prime} \leqq 3 / 2$ in $d=2$.

\section{Appendix: On the Independence of the Invasion Pattern from the Starting Set}

Here we show that in $d=2$, the infinite-time invaded regions that evolve from distinct, finite starting sets differ at only a finite number of bonds, with probability one. This is a strong form of independence from initial conditions. Indeed, one can envision weaker forms in which the number of differing bonds grows with $n$ in such a way that the fraction of differing bonds still tends to zero. Although our techniques do not extend to $d \geqq 3$, one might suppose that some form of independence holds in higher dimensions. It should be noted, however, that a proof that the infinite-time invaded regions evolving from distinct starting sets are nondisjoint, with probability one, would imply as a corollary the uniqueness for any $p$ of infinite clusters in Bernoulli percolation, an as yet unproved result for $d \geqq 3$ (see [9]).

Independence of the invasion pattern from the starting set is in contrast to what is expected for certain other stochastic growth models. For example, diffusion limited aggregation seems to have a strong dependence on initial conditions, as indicated by an epicenter (e.g., discharge point) in the numerically generated configurations [21-24]. That typical invasion configurations (at least in $d=2$ ) are essentially homogeneous is the content of the following.

Theorem A.1. Let $U, V \subset \mathbb{B}_{2}$ be finite starting sets for the invasion process on $\mathbb{B}_{2}$, and denote by $\mathbf{C}^{[U]}(\Gamma)$ and $\mathbf{C}^{[V]}(\Gamma)$ the corresponding infinite-time invaded regions in the configuration $\Gamma \in[0,1]^{\mathbb{B}_{2}}$. Then, with probability one, the sets $\mathbf{C}^{[U]}(\Gamma)$ and $\mathbf{C}^{[V]}(\Gamma)$ differ at only a finite number of bonds, i.e.

$$
\left|\mathbf{C}^{[U]}(\Gamma) \Delta \mathbf{C}^{[V]}(\Gamma)\right|<\infty \text { with probability } 1 .
$$

Here $\Delta$ denotes the symmetric difference of the sets

$$
\mathbf{C}^{[U]}(\Gamma) \Delta \mathbf{C}^{[V]}(\Gamma) \equiv \mathbf{C}^{[U]}(\Gamma) \cup \mathbf{C}^{[V]}(\Gamma) \backslash \mathbf{C}^{[U]}(\Gamma) \cap \mathbf{C}^{[V]}(\Gamma) .
$$

Moreover, denoting by $\mathbf{C}_{n}^{[U]}(\Gamma)$ and $\mathbf{C}_{n}^{[V]}(\Gamma)$ the invaded regions at time $n$,

$$
\sup _{n}\left|\mathbf{C}_{n}^{[U]}(\Gamma) \Delta \mathbf{C}_{n}^{[V]}(\Gamma)\right|<\infty \text { with probability } 1 \text {. }
$$

Proof. Let $U, V \subset \mathbb{B}_{2}$ be two finite starting sets. Let $\Gamma \in[0,1]^{\mathbb{B}_{2}}$ and denote by $\Gamma(b)$ the value that $\Gamma$ assigns to the bond $b \in \mathbb{B}_{2}$. As in the proofs of Theorems 3.5 and 4.3, we can map $\Gamma$ into a $y$ density percolation configuration by identifying the bond $b$ as "occupied" if $\Gamma(b) \leqq y$. Here we choose $y=p_{c}$, so that our identification 


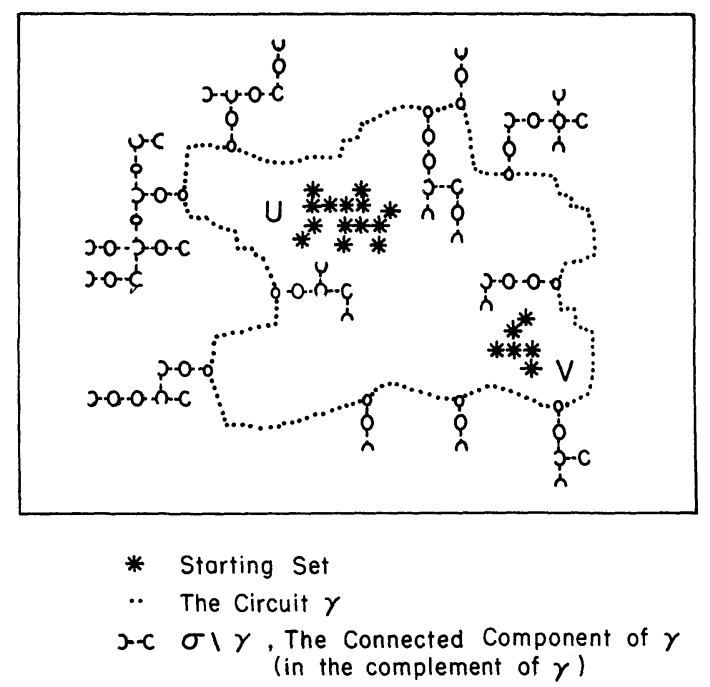

Fig. 2. Innermost circuit and its connected component

maps $\Gamma$ into a Bernoulli configuration at threshold. It then follows from the Harris [25] and Kesten [6] theorems that, with probability one, there are infinitely many circuits of these occupied bonds surrounding $U \cup V$.

Let $\gamma$ be any circuit of bonds (not necessarily occupied) surrounding $U \cup V$. We denote by $G_{\gamma}$ the event that $\gamma$ is the innermost circuit of occupied bonds surrounding $U \cup V$. Note that the $G_{\gamma}$ form a disjoint partition of $p_{c}$ density Bernoulli configurations, and thus, by our identification, a disjoint partition of $[0,1]^{\mathbb{B}_{2}}$ (provided that we include the zero probability event $G_{\emptyset}$ that $U \cup V$ is not surrounded by an occupied circuit).

Let us partition the set $[0,1]^{\mathbb{B}_{2}}$ even further. To this end, note that if we are given a $\Gamma \in G_{\gamma}$, we can determine the set of occupied bonds which form the connected component of $\gamma$ (see Fig. 2). With probability one, this set is finite. In any case, we can consider all possible connected components of $\gamma$, and index them by $\sigma$. We denote by $G_{\gamma, \sigma} \subset G_{\gamma} \subset[0,1]^{\mathbb{B}_{2}}$ the sub-event of $G_{\gamma}$ that $\sigma$ is the connected component of occupied bonds of $\gamma$. The $G_{\gamma, \sigma}$ form a (finer) disjoint partition of $[0,1]^{\mathbb{B}_{2}}$.

For any configuration $\Gamma \in[0,1]^{\mathbb{B}_{2}}$, we may define the set $S(\Gamma) \subset \mathbb{B}_{2}$ by

$$
S(\Gamma) \equiv\{b \in \sigma\} \cup\left\{b \in \gamma^{0}\right\}
$$

for the unique $\sigma, \gamma$ for which $\Gamma \in G_{\gamma, \sigma}$. Here $\gamma^{0}$ denotes the set of all bonds which lie inside $\gamma$. Again we note that, with probability one, $|S(\Gamma)|<\infty$.

Now let us return to the question at hand. Given $\Gamma \in[0,1]^{\mathbb{B}_{2}}$, it suffices to show that $\left|\mathbf{C}_{n}^{[U]}(\Gamma) \Delta \mathbf{C}_{n}^{[V]}(\Gamma)\right|$ is bounded above uniformly in $n$.

To this end, let us define the sets

and

$$
\mathbf{C}_{n, S}^{[U]}(\Gamma) \equiv \mathbf{C}_{n}^{[U]}(\Gamma) \cap S(\Gamma)
$$

$$
\mathbf{C}_{n, S^{c}}^{[U]}(\Gamma) \equiv \mathbf{C}_{n}^{[U]}(\Gamma) \backslash S(\Gamma),
$$


and similarly for the $\mathbf{C}_{n}^{[V]}(\Gamma)$. Thus $\mathbf{C}_{n, S}^{[U]}(\Gamma)$ represents the part of the invaded region which lies within the innermost occupied circuit or overlaps its connected component, and $\mathbf{C}_{n, S^{c}}^{[U]}(\Gamma)$ is the rest of the invaded region [i.e., that part in $S^{c}(\Gamma)$ $\left.\equiv \mathbb{B}_{2} \backslash S(\Gamma)\right]$. We have

$$
\left|\mathbf{C}_{n}^{[U]}(\Gamma) \Delta \mathbf{C}_{n}^{[V]}(\Gamma)\right|=\left|\mathbf{C}_{n, S}^{[U]}(\Gamma) \Delta \mathbf{C}_{n, S}^{[V]}(\Gamma)\right|+\left|\mathbf{C}_{n, S c}^{[U]}(\Gamma) \Delta \mathbf{C}_{n, S c}^{[V]}(\Gamma)\right| .
$$

The first term in (A.3) is easy to handle. We use the (ridiculous) bound:

$$
\left|\mathbf{C}_{n, S}^{[U]}(\Gamma) \Delta \mathbf{C}_{n, S}^{[V]}(\Gamma)\right| \leqq|S(\Gamma)| .
$$

The key observation in bounding the second term of (A.3) is the following: The same bonds of $S^{c}(\Gamma)$ will be invaded by regions with starting sets $U$ and $V$. Moreover, these bonds will be invaded in the same order. Note that the second statement does not mean that if a given bond $b \in S^{c}(\Gamma)$ is invaded on the $j^{\text {th }}$ step by the $U$-cluster, then it will also be invaded on the ${ }^{t \text { th }}$ step by the $V$-cluster. Rather, it means that if $b \in S^{c}(\Gamma)$ is the $j^{\text {th }}$ bond of $S^{c}(\Gamma)$ to be invaded by the $U$-cluster, then it will also be the $j^{\text {th }}$ bond of $S^{c}(\Gamma)$ to be invaded by the $V$-cluster. The conclusion is that, at any time $n$, either $\mathbf{C}_{n, S^{c}}^{[U]}(\Gamma) \subset \mathbf{C}_{n, S^{c}}^{[V]}(\Gamma)$ or $\mathbf{C}_{n, S^{c}}^{[V]}(\Gamma) \subset \mathbf{C}_{n, S^{c}}^{[U]}(\Gamma)$. This, however, implies

$$
\left|\mathbf{C}_{n, S^{c}}^{[U]}(\Gamma) \Delta \mathbf{C}_{n, S^{c}}^{[V]}(\Gamma)\right|=|| \mathbf{C}_{n, S^{c}}^{[U]}(\Gamma)|-| \mathbf{C}_{n, S^{c}}^{[V]}(\Gamma)|| .
$$

Now note that $\left|\mathbf{C}_{n, S^{c}}^{[U]}(\Gamma)\right|$, which is simply the number of bonds in $S^{c}(\Gamma)$ invaded by the $U$-cluster by time $n$, has the obvious bounds

$$
n-|S(\Gamma)| \leqq\left|\mathbf{C}_{n, S^{c}}^{[U]}(\Gamma)\right| \leqq n,
$$

and similarly for $\left|\mathbf{C}_{n, S^{c}}^{[V]}(\Gamma)\right|$. Combining this with Eq. (A.5), we have

$$
\left|\mathbf{C}_{n, S^{c}}^{[U]}(\Gamma) \Delta \mathbf{C}_{n, S^{c}}^{[V]}(\Gamma)\right| \leqq|S(\Gamma)| .
$$

It then follows from (A.3), (A.4), and (A.7) that

$$
\left|\mathbf{C}_{n}^{[U]}(\Gamma) \Delta \mathbf{C}_{n}^{[V]}(\Gamma)\right| \leqq 2|S(\Gamma)| .
$$

With probability one, the ( $n$-independent) upper bound is finite.

Acknowledgements. Two of us (J. T. C. and L. C.) thank H. J. Weismann for a useful discussion, and for showing us his numerical simulations of invasion percolation and some related processes. J. T. C. and L. C. also thank the National Science Foundation for its support and the opportunities this has provided. One of us (C. M. N.) thanks the John S. Guggenheim Memorial Foundation and the National Science Foundation for their support.

\section{References}

1. Lenormand, R., Bories, S.: C.R. Acad. Sci. 291, 279 (1980)

2. Chandler, R., Koplick, J., Lerman, K., Willemsen, J.F.: J. Fluid Mech. 119, 249 (1982)

3. Chayes, J.T., Chayes, L., Newman, C.M.: The stochastic geometry of invasion percolation. II. Trapping and the external surface transition (in preparation)

4. Wilkinson, D., Willemsen, J.F.: Invasion percolation: A new form of percolation theory. J. Phys. A: Math. Gen. 16, 3365 (1983)

5. Nickel, B., Wilkinson, D.: Invasion percolation on the Cayley tree: exact solution of a modified percolation model. Phys. Rev. Lett. 51, 71 (1983) 
6. Kesten, $\bar{H}$.: The critical probability of bond percolation on the square lattice equals $\frac{1}{2}$. Commun. Math. Phys. 74, 41 (1980)

7. Russo, L.: Z. Wahrscheinlichkeitstheor. Verw. Geb. 56, 229 (1981)

8. Kesten, H.: Percolation theory for mathematicians. Boston: Birkhäuser 1982

9. Newman, C.M., Schulman, L.S.: Infinite clusters in percolation models. J. Stat. Phys. 26, 613 (1981)

10. Kunz, H., Souillard, B.: Essential singularity in percolation problems and asymptotic behavior of cluster size distribution. J. Stat. Phys. 19, 77 (1978)

11. Aizenman, M., Deylon, F., Souillard, B.: Lower bounds on the cluster size distribution. J. Stat. Phys. 23, 267 (1980)

12. Russo, L.: Z. Wahrscheinlichkeitstheor. Verw. Geb. 43, 39 (1978)

13. Seymour, P.D., Welsh, D.J.A.: Ann. Discrete Math. 3, 227 (1978)

14. Aizenman, M., Newman, C.M.: Tree graph inequalities and critical behavior in percolation models. J. Stat. Phys. 36, 107 (1984)

15. Hammersley, J.M.: Ann. Math. Stat. 28, 790 (1957)

16. Kesten, H.: Analyticity properties and power law estimates of functions in percolation theory. J. Stat. Phys. 25, 717 (1981)

17. Aizenman, M., Newman, C.M.: In preparation

18. Aizenman, M., Chayes, J.T., Chayes, L., Fröhlich, J., Russo, L.: On a sharp transition from area law to perimeter law in a system of random surfaces. Commun. Math. Phys. 92, 19 (1983)

19. Campanino, M., Russo, L.: An upper bound on the critical probability for the threedimensional cubic lattice. Ann. Probab. (in press)

20. van den Berg, J., Kesten, H.: Inequalities with applications to percolation and reliability, preprint (1984)

21. Witten, T.A., Sander, L.M.: Diffusion-limited aggregation, a kinetic critical phenomenon. Phys. Rev. Lett. 47, 1400 (1981)

22. Witten, T.A., Sander, L.M.: Phys. Rev. B 27, 5686 (1983)

23. Meakin, P.: Diffusion-controlled cluster formation in 2-6-dimensional space. Phys. Rev.A27, 1495 (1983)

24. Niemeyer, L., Pietronero, L., Wiesmann, H.J.: Fractal dimension of dielectric breakdown. Phys. Rev. Lett. 52, 1033 (1984)

25. Harris, T.E.: Proc. Cambridge Philos. Soc. 56, 13 (1960)

26. Grimmett, G.: Lond. Math. Soc. (2) 23, 372 (1981)

27. Wilkinson, D., Barsony, M.: J. Phys. A: Math. Gen. 17, L 129 (1984)

28. Hammersley, J.M.: A Monte Carlo solution of percolation in the cubic lattice. In: Methods in computational physics, Vol.I. New York: Academic Press 1963

Communicated by A. Jaffe

Received January 7, 1985; in revised form April 22, 1985

Note added in proof. We have recently obtained results concerning the large-time asymptotics and the finite cluster distribution, $P_{n}(y)$, which significantly improve the results of Sect.6. In particular, we have shown that

$$
\lim _{n \rightarrow \infty} \log \log \left(1 / P_{n}(y)\right)=(d-1) / d
$$

for $y$ above a limit of slap thresholds. This and related theorems can be found in J.T. Chayes, L. Chayes, and C.M. Newman: Bernoulli percolation above threshold: An invasion percolation analysis, IHES preprint. 
